\title{
Electronic Patient-Reported Outcomes After Thoracic Surgery: Toward Better Remote Management of Perioperative Symptoms
}

\author{
Cecilia Pompili, MD, $\mathrm{PhD}^{1,2}$ (1) , Ethan Basch, MD, $\mathrm{MSc}^{3,4}$, Galina Velikova, $\mathrm{MD}, \mathrm{PhD}^{\mathbf{1}}$, and \\ Gita N. Mody, MD, MPH ${ }^{3,5}$
}

${ }^{1}$ Section of Patient-Centred Outcomes Research, Leeds Institute for Medical Research at St James's, University of Leeds, Leeds, UK; ${ }^{2}$ Leeds Teaching Hospital Trust, Leeds, UK; ${ }^{3}$ Lineberger Comprehensive Cancer Center, University of North Carolina, Chapel Hill, NC; ${ }^{4}$ Division of Oncology, University of North Carolina, Chapel Hill, NC; ${ }^{5}$ Department of Surgery, University of North Carolina, Chapel Hill, NC

Health-related quality of life (HRQOL) after thoracic surgery is increasingly important due to steadily improving survival and better clinical outcomes after treatment for early-stage lung cancer. The recent article by Avery and colleagues $^{1}$ describes HRQOL in patients undergoing video-assisted thoracoscopic surgery (VATS) for lung cancer in a single UK center. This prospective, longitudinal study is one of the few to use validated, disease-specific survey instruments to study this population., ${ }^{2,3}$ Until the results of the UK-based VIOLET trial (comparing the impact of VIdeo assisted thoracoscopic lobectomy versus conventional Open LobEcTomy) are available, ${ }^{4}$ this study provides the most contemporary information on HRQOL trajectory after lung cancer surgery. Importantly, the authors show HRQOL continues to suffer and residual symptoms persist at 1 year after minimally invasive lung cancer surgery. These findings demonstrate the need for longitudinal measurement of postoperative patient-reported outcomes (PROs) in order to (1) provide an adjunct to traditional clinical outcomes as measures of quality, and (2) improve symptom management in routine clinical practice. Given the heterogeneity of international guidelines regarding the optimal follow-up schedule and duration after thoracic surgery, ${ }^{5}$ electronic PRO (ePRO) platforms

\section{(C) Society of Surgical Oncology 2021}

First Received: 27 October 2020

Accepted: 11 December 2020;

Published Online: 21 January 2021

C. Pompili, MD, $\mathrm{PhD}$

e-mail: c.pompili@leeds.ac.uk that automatically deliver PRO surveys to patients at home and alert providers for concerning responses may serve to effectively monitor patients beyond the standard post-surgical follow-up periods. ${ }^{6}$

From the article by Avery and colleagues, we now have evidence that collecting HRQOL data is feasible before and after lung cancer resection. The trajectory of HRQOL allows us to broaden understanding of treatment effects on patients, enabling an informed shared preoperative decision-making process. Of note, the authors achieved impressively high survey response rates, perhaps owing to their status as a pioneering enhanced recovery after surgery (ERAS) programme center. Engaging clinicians and integrating ePRO platforms into current clinical workflows such as ERAS has already been demonstrated to be a critical aspect of the success of routine collection of PROs ${ }^{7}$ and may promote the use of ePROs in reducing postoperative symptom burden. ${ }^{8}$ Indeed, there was a very low attrition rate from the study $(76.6 \%$ of patients returned their 12-month questionnaire), suggesting that the longitudinal collection of perioperative PROs is possible.

These findings demonstrate the critical need to develop programs to pre-emptively address symptom burden and QOL deficits in lung resection patients. In the last decades, there has been growing interest in the measurement of PROs and their integration into electronic health records for use in clinical care improvement, ${ }^{9}$ as well as in quality improvement databases as metrics of performance. Symptom monitoring during cancer treatment via web-based ePROs has been demonstrated to lengthen survival, ${ }^{10}$ leading to implementation of ePRO systems in many oncological settings and pilot trials in the surgical field. ${ }^{11}$ 
Despite the potential benefits of integrating ePROs into routine clinical care, there are clear barriers that need to be overcome, and several international societies have released guidance to address these. ${ }^{12,13}$ However, in the last 6 months, the coronavirus disease 2019 (COVID-19) pandemic has revolutionized perioperative care, forcing more remote contacts and video or telephone consultations. ${ }^{14}$ This portends the potential application of remote ePRO software systems postoperatively, which allows patients to report symptoms from home and receive immediate advice on the self-management of mild symptoms and recommendations for contacting general practitioners or the hospital for severe symptoms. ${ }^{6,15}$

It is clear that incorporation of ePROs into thoracic surgery practice is necessary to modernize our approach to outcomes assessment and improvement. This pandemic has even further raised the need for our patients to be safely followed from home, particularly since COVID-19 is a respiratory virus, leading to concern on how to discriminate its symptoms from the normal post-lobectomy recovery. The pandemic has also forced the elderly and other demographically challenged populations to be more facile with remote follow-ups, eliminating one barrier to ePRO use. ePROs may allow us to standardize and streamline a postoperative process, simultaneously reducing hospital visits, improving surgical outcomes, and avoiding the associated COVID-19 infection risk to in-person care.

Efforts should now be focused on the implementation of routine remote ePRO monitoring as part of the peri-surgical pathway. One of the main barriers in implementing ePRO is the clinicians' knowledge to meaningfully interpret and integrate PRO data into their clinical practice ${ }^{16}$; therefore, research such as this from Avery and colleagues is pivotal for benchmarking and in addressing these issues. Ultimately, the potential use of ePROs collected for routine individual monitoring will also help in evaluating and improving thoracic surgery service quality on a population level.

DISCLOSURE Ethan Basch receives research funding from the US National Cancer Institute and Patient-Centered Outcomes Research Institute, and is an advisor to Sivan, CareVive, AstraZeneca, and Navigating Cancer. Galina Velikova receives personal fees from Roche, Eisai, and Novartis, and grants from Breast Cancer Now, EORTC, Yorkshire Cancer Research, and Pfizer. Cecilia Pompili and Gita N. Mody have no conflicts of interest to report.

\section{REFERENCES}

1. Avery KNL, Blazeby JM, Chalmers KA, Batchelor TJP, Casali $\mathrm{G}$, Internullo $\mathrm{E}$, et al. Impact on health-related quality of life of video-assisted thoracoscopic surgery for lung cancer. Ann Surg Oncol. 2020;27(4):1259-71.

2. Pompili C, Brunelli A, Xiume F, Refai M, Salati M, Sabbatini A. Predictors of postoperative decline in quality of life after major lung resections. Eur J Cardio-Thorac Surg. 2011;39(5):732-7.

3. Bendixen M, Jørgensen OD, Kronborg C, Andersen C, Licht PB. Postoperative pain and quality of life after lobectomy via videoassisted thoracoscopic surgery or anterolateral thoracotomy for early stage lung cancer: a randomised controlled trial. Lancet Oncol. 2016;17(6):836-44.

4. Lim E, Batchelor T, Shackcloth M, Dunning J, McGonigle N, Brush T, et al. Study protocol for video assisted thoracoscopic lobectomy versus conventional Open LobEcTomy for lung cancer, a UK multicentre randomised controlled trial with an internal pilot (the VIOLET study). BMJ Open. 2019;9(10):e029507.

5. Pompili C, Edwards M, Bhandari P, Novoa N, Hasegawa S, Yoshino I, et al. STS, ESTS and JACS survey on surveillance practices after surgical resection of lung cancer. Interact Cardiovasc Thorac Surg. 2019;29(4):532-8.

6. Basch E, Mody GN, Dueck AC. Electronic patient-reported outcomes as digital therapeutics to improve cancer outcomes. JCO Oncol Pract. 2020;16(9):541-2.

7. Medbery RL, Fernandez FG, Khullar OV. ERAS and patient reported outcomes in thoracic surgery: a review of current data. $J$ Thorac Dis. 2019;11 Suppl 7:S976-86.

8. Cleeland CS, Wang XS, Shi Q, Mendoza TR, Wright SL, Berry $\mathrm{MD}$, et al. Automated symptom alerts reduce postoperative symptom severity after cancer surgery: a randomized controlled clinical trial. J Clin Oncol. 2011;29(8):994-1000.

9. Velikova G, Brown JM, Smith AB, Selby PJ. Computer-based quality of life questionnaires may contribute to doctor-patient interactions in oncology. Br J Cancer. 2002;86(1):51-9.

10. Denis F, Basch E, Septans A-L, Bennouna J, Urban T, Dueck AC, et al. Two-year survival comparing web-based symptom monitoring vs routine surveillance following treatment for lung cancer. JAMA. 2019;321(3):306-7.

11. Khullar OV, Rajaei MH, Force SD, Binongo JN, Lasanajak Y, Robertson S, et al. Pilot study to integrate patient reported outcomes after lung cancer operations into the society of thoracic surgeons database. Ann Thorac Surg. 2017;104(1):245-53.

12. Gensheimer SG, Wu AW, Snyder CF. Oh, the places we'll go: patient-reported outcomes and electronic health records. Patient. 2018;11(6):591-8.

13. Basch E, Barbera L, Kerrigan CL, Velikova G. Implementation of patient-reported outcomes in routine medical care. Am Soc Clin Oncol Educ Book. 2018;38:122-34.

14. Schrag D, Hershman DL, Basch E. Oncology practice during the COVID-19 pandemic. JAMA. 2020;323(20):2005-6.

15. Absolom K, Holch P, Warrington L, Samy F, Hulme C, Hewison $\mathrm{J}$, et al. Electronic patient self-reporting of adverse-events: patient information and advice (eRAPID): a randomised controlled trial in systemic cancer treatment. BMC Cancer. 2017;17(1):318.

16. Nguyen H, Butow P, Dhillon H, Sundaresan P. A review of the barriers to using patient-reported outcomes (PROs) and patientreported outcome measures (PROMs) in routine cancer care. $J$ Med Radiat Sci. Epub 19 Aug 2020. https://doi.org/10.1002/jmrs. 421.

Publisher's Note Springer Nature remains neutral with regard to jurisdictional claims in published maps and institutional affiliations. 